- researchers are grappling with involves diseased heart valves. Bacteria such as $S$. aureus can enter the bloodstream as a result of practices such as needle sharing or not cleaning the skin before injecting a drug. If the infection reaches the heart, it can damage the valves. Severe cases can require a heart transplant.

In an ongoing study, microbiologist Cecilia Thompson at the University of North Carolina in Chapel Hill is sequencing DNA from heart valves collected from people who have had surgery to replace diseased valves with artificial ones. Thompson has found that valves taken from people who had injected drugs are more likely to be infected with $S$. aureus than are those of people who don't use drugs.

Thompson presented her results at the American Society for Microbiology meeting in San Francisco, California, on 21 June. But these are just the latest observations of what seems to be a worrying trend. In a study published in January ${ }^{1}$, researchers found a 13 -fold increase in heart infections among drug users in North Carolina between 2007 and 2017. Until 2013, surgeons in the state performed fewer than 10 operations annually to treat drug-related heart infections, compared with more than 100 in 2017.

Opioids themselves - rather than the method used to inject them - could also be making people more susceptible to infection.
Another study ${ }^{2}$, also published in January, looked at more than 25,000 people treated at veterans' health facilities between 2000 and 2012. It found that people who took medium or high doses of prescribed opioids for pain management were significantly more susceptible to pneumonia. It's unclear why, but research in monkeys suggests that some prescription opioids, such as morphine, suppress the immune system ${ }^{3}$.

\section{IT'S ABOUT RELATIONSHIPS}

Researchers also struggle with anticipating the location of outbreaks associated with drug use, because the patterns can differ from those of non-drug-related ones.

But a computer model developed by Georgiy Bobashev, a data scientist at RTI International, a non-profit research institute in Research Triangle Park, North Carolina, and his colleagues simulates drug users and their social networks to predict where outbreaks of HIV infections might occur. The program considers factors including whom users know, the type of heroin available to them - which could affect the presence of pathogens - and their experience with the drug.

The social component of predicting outbreak patterns is crucial, Bobashev says. People who used drugs during the height of the HIV epidemic in the 1990s learnt safe injection practices, he says, but newer users are more likely to use riskier methods, such as sharing needles.

In an unpublished analysis, Bobashev's group's model predicted that HIV outbreaks related to opioids would be concentrated in small geographic pockets, rather than spread over a wider area, as researchers would expect with non-drug-related outbreaks.

Data from real life bolster this result. Previous opioid-related HIV outbreaks, including one in 2014 in Scott County, Indiana, followed this pattern. And in March, the West Virginia health department announced an outbreak in Cabell County due to a spike in new cases of HIV acquired through drug use.

The key to stopping the rise in opioidassociated infections and halting their rise is to treat opioid use as a disease without stigmatizing drug users, says Carlos Del Rio, a global-health researcher at Emory University in Atlanta, Georgia.

A working group at the US National Academy of Medicine, which Del Rio is leading, has started to develop a strategy for integrating care for infections and opioid use. "The opioid epidemic is going to be to [young medical students] what HIV was to me," Del Rio says. "You'd better get used to it." -

1. Schranz, A. J., Fleischauer, A., Chu, V. H., Wu, L.-T. \& Rosen, D. L. Ann. Intern. Med. 170, 31-40 (2019).

2. Edelman, E. J. et al. JAMA Intern. Med. 179, 297-304 (2019).

3. Kumar, R. et al. J. Virol. 78, 11425-11428 (2004).

\title{
Discrimination drives LGBT+ scientists to think about quitting
}

\section{Physical scientists from sexual and gender minorities are excluded at work, finds UK survey.}

\section{BY ELIZABETH GIBNEY}

$\mathrm{N}$ early one-third of physical scientists from sexual and gender minorities in the United Kingdom have considered leaving their jobs because of their workplace climate, a survey suggests.

Some $18 \%$ of these LGBT+ scientists said they had experienced harassment, bullying or exclusionary behaviour in the workplace. That figure rises to $32 \%$ for transgender people and those who don't identify as either male or female (non-binary). Transgender and non-binary scientists were overall more likely than other the groups to describe challenges in their work environment, and women generally reported more negative experiences than men.

The findings come from a survey published on 26 June by British scientific societies that spoke to more than 600 people working in

\section{LGBT+ EXPERIENCES}

In a UK survey of LGBT+ physical scientists, non-binary and transgender people were most likely to feel uncomfortable at work and to experience exclusionary behaviour.

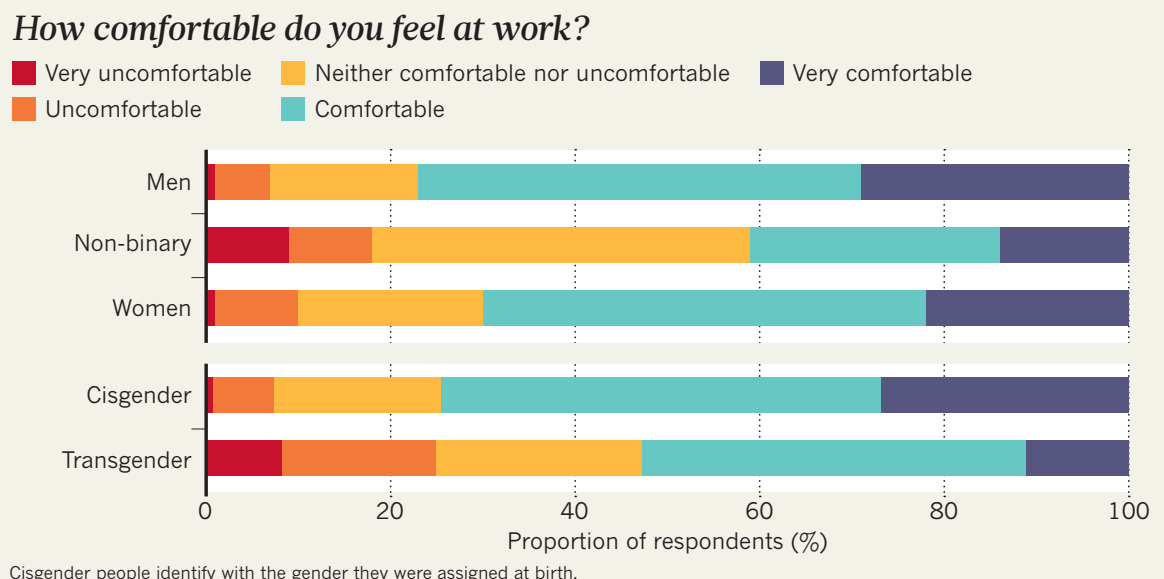


$\stackrel{\sim}{\alpha}$ academia, industry and schools - the largest such study worldwide in the physical or sciences (see 'LGBT+ experiences'). Most respondents self-identified as LGBT+, although the survey also included a minority of people who were both heterosexual and cisgender - identifying with the gender they were assigned at birth

Language - such as derogatory use of the word gay - harmful humour and people using the wrong pronouns were some of the factors that created an unwelcoming climate. It's "all the tiny things that, over time, build up to create a culture that isn't particularly welcoming", says Jennifer Dyer, head of diversity at the London-based Institute of Physics, which conducted the survey with the Royal Astronomical Society and the Royal Society of Chemistry.

Overall, three-quarters of LGBT+ respondents reported feeling comfortable at work and almost 70\% said they thought the situation was improving. But this masks "an underlying picture", in which almost half agreed there was a lack of awareness of LGBT+ issues at work, says Dyer.

\section{REPRESENTATION MATTERS}

The results echo those of a survey published by the American Physical Society in 2016, and come as the academic community grapples with endemic discrimination against and exclusion of minority communities. The latest survey is crucial as a benchmark against which to monitor progress, says astrophysicist Alfredo Carpineti, who cofounded the UK campaign organization Pride in STEM (science, technology, engineering and mathematics) in 2016.

The report says that despite a wealth of evidence that diversity benefits science, workplaces still have much to do to cultivate inclusive environments. The societies make specific recommendations for individuals and institutions, including championing LGBT+ scientists and networks, and implementing science-specific training that effectively supports LGBT+ staff. It also suggests that people put their pronouns in e-mail signatures and on name badges at events. This normalizes the idea that not everybody is a he or a she, says Dyer.

One reason that scientists might face more challenges than people in other sectors is the international nature of research - a successful career often means interacting with people in cultures that are less inclusive of LGBT+ people. Scientists reported feeling less safe being open about their gender or sexual identity when working in these cultures, and in some cases felt they might be expected to "return to the closet" for the sake of a smooth collaboration. It is essential that institutions reflect the international nature of science in their LGBT+ policies, says the report. a SEE EDITORIALP.5

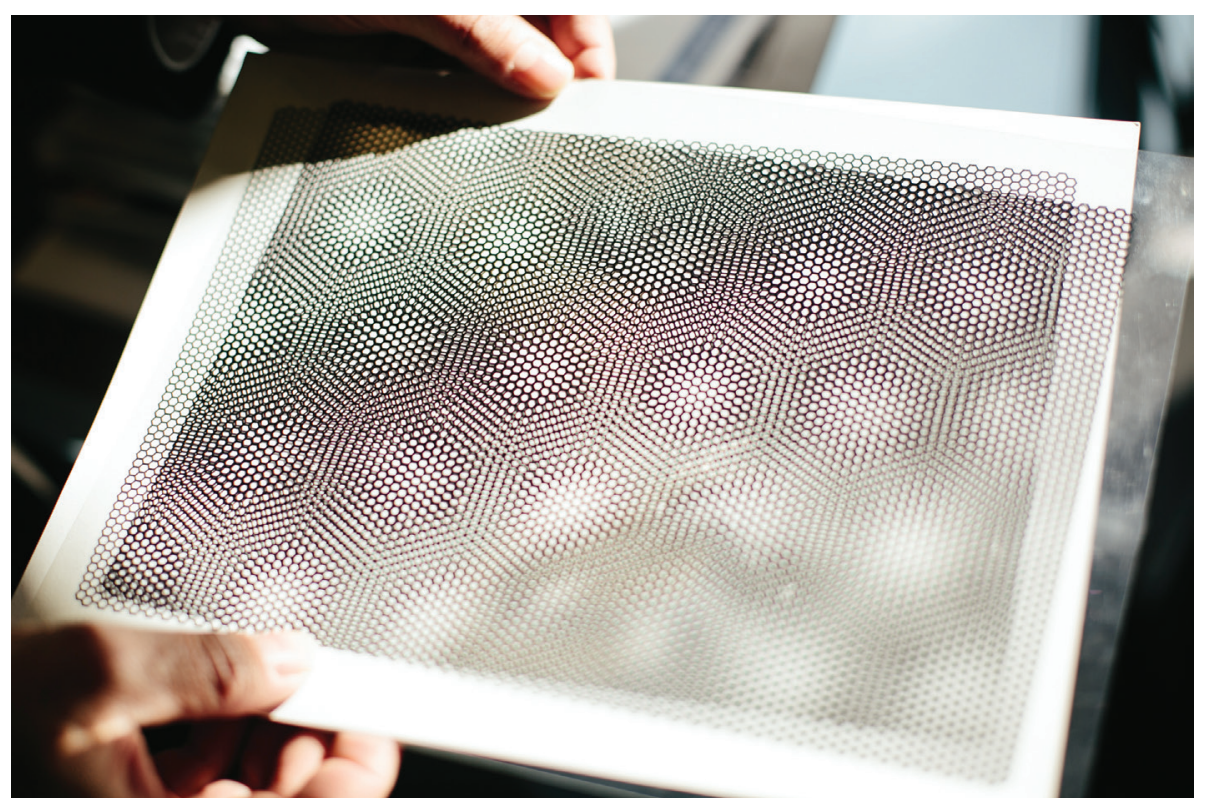

Misaligned layers of graphene seem to exhibit a phenomenon known as fragile topology.

TOPOLOGY

Strange materials
excite physicists

'Fragile topology' is the latest addition to a set of quantum phenomena that give materials exotic properties.

\section{BY DAVIDE CASTELVECCHI}

$\mathrm{T}$ The mathematics hidden in materials keeps getting more exotic. Topological states of matter - which derive exotic properties from their electrons' 'knotty' quantum states - have shot from rare curiosity to one of the hottest fields in physics. Now, theorists are finding that topology is ubiquitous - and are recognizing it as one of the most significant ways in which solid matter can behave.

In the past few years, physicists have identified a 'fragile' version of topology that might occur in almost all crystalline solids, according to a preprint published in May ${ }^{1}$. Another study ${ }^{2}$, published last month, describes hints of a fragile state in the electrons of a carbon-based device - which could be the first experimental evidence for fragile topology.

It is too early to say whether these discoveries will have a major impact on practical materials. But researchers suggest it might help to explain certain kinds of superconductivity, and could be important in photonics - technologies that carry information in light pulses rather than electrons.

The latest studies show that fragile topology "is not just a radical, academic rabbit hole that people are going down", says Ashvin Vishwanath, a theoretical physicist specializing in condensed matter at Harvard University in Cambridge, Massachusetts. "I am having a hard time keeping up with the field, even though it's just a year old."

Topology is the branch of mathematics that deals with deformations that reshape objects continuously - as opposed to those that cut or break objects, in the way that cutting two linked loops unlinks them. In some materials, electrons can exist in 'knotty' quantum states, and these can keep an electron moving in a particular direction, because altering course would require an abrupt change of its state, akin to cutting a knot. As a result, the physical qualities are protected, allowing some materials called topological insulators to be, despite their names, perfect conductors on their outer edges while the bulk of the material is insulating.

'Strongly topological' materials that harbour these robust effects might provide the basis of future topological quantum computers, which could solve certain problems exponentially faster than classical computers.

Strong topological properties come from quirks in the quantum states of electrons: rather than crowding around individual $>$ 